\title{
Plus-strand DNA Synthesis of the Yeast Retrotransposon Ty1 is Initiated at Two Sites, PPT1 Next to the 3' LTR and PPT2 Within the pol Gene. PPT1 is Sufficient for Ty1 Transposition
}

\author{
T. Heyman ${ }^{1 \star}$, B. Agoutin', S. Friant ${ }^{2}$, F. X. Wilhelm² and M. L. Wilhelm²*
}

${ }^{1}$ Unité de Recherche Associée 1342 du Centre National de la Recherche Scientifique Institut Curie-Biologie Centre Universitaire 91405 Orsay Cedex, France

${ }^{2}$ Unité Propre de Recherche 9002 du Centre National de la Recherche Scientifique

Institut de Biologie Moléculaire et Cellulaire 15, rue René Descartes 67084 Strasbourg

Cedex, France
Long terminal repeat elements and retroviruses require primers for initiation of minus and plus-strand DNA synthesis by reverse transcriptase. Here we demonstrate genetically that plus-strand DNA synthesis of the yeast Ty1 element is initiated at two sites located at the $5^{\prime}$ boundary of the $3^{\prime}$ long terminal repeat (PPT1) and near the middle of the pol gene in the integrase coding sequence (PPT2). A consequence of the presence of two PPTs is that Ty1 plus-strand DNA exists as segments at some time during replication. Three fragments have been identified: the plus-strand strong-stop DNA initiated at PPT1, a downstream fragment initiated at PPT2 and an upstream fragment spanning the 5 '-terminal part of Ty1 and a portion of the TyB gene. Characterization of the 3 ' ends of the plus-strand DNA fragments reveals (1) that the upstream fragment is elongated beyond PPT2 creating a plus-strand overlap and (2) that the majority of plus-strand strong-stop DNA fragments bear a copy of the minus-strand primer binding site in agreement with the accepted model of retroviral genomic RNA reverse transcription.

The two polypurine tracts, PPT1 and PPT2, have an identical sequence GGGTGGTA. Mutations replacing purines by pyrimidines in this sequence significantly diminish or abolish initiation of plus-strand synthesis. Ty1 elements bearing a mutated PPT2 sequence are not defective for transposition whereas mutations in PPT1 abolish transposition.

(C) 1995 Academic Press Limited

Keywords: retrotransposon; yeast; reverse transcription; 3' end termination; plus-strand primers
U3-R. Reverse transcription of RNA generates a complete double-stranded DNA molecule with two LTRs which is able to integrate into the nuclear genome (for a review see Boeke \& Sandmeyer, 1991).

Most LTR-retroelements and retroviruses use specific primers to initiate minus and plus-strand cDNA synthesis. Minus-strand cDNA synthesis starts near the 5' end of the genomic RNA using a base-paired cellular tRNA as a primer whereas plus-strand cDNA synthesis commences from an RNaseH-resistant oligoribonucleotide spanning a purine-rich sequence (polypurine tract, PPT). In some retroviruses, characterization of the subgenomic plus-strand fragments has shown that several plus-strand priming sites can be used by reverse transcriptase. For HIV-1, plus-strand DNA synthesis is primed by two copies of the PPT, one adjacent to U3 ( 3 ' end PPT) and the other located near the centre of the genome within the pol gene (Charneau \& Clavel, 1991). Introduction of mutations replacing 
purine residues by pyrimidine residues in the central PPT significantly slows down viral growth suggesting that this central PPT is important for efficient HIV-1 replication (Charneau et al., 1992).

Replication of the yeast LTR-retrotransposons occurs by a mechanism similar to that of retroviruses. Ty1 genomic RNA is packaged into intracytoplasmic nucleoprotein complexes called virus-like particles (VLPs). Reverse transcription of the RNA into double-stranded DNA takes place in these particles (Eichinger \& Boeke, 1988). As in the case of retroviruses, the synthesis of the Ty1 minus-strand cDNA is primed by a cellular tRNA hybridized to the genomic RNA at the primer binding site (PBS) located $3^{\prime}$ to the U5 region (Chapman et al., 1992). The Ty1 minus-strand primer is the methionine initiator tRNA packaged into the VLPs together with the genomic RNA (Chapman et al., 1992; Pochart et al., 1993a). Two putative primers for plus-strand cDNA synthesis have been identified in Ty1: PPT1 located at the 5' boundary of the 3' long terminal repeat and PPT2 near the middle of the pol gene in the integrase coding sequence. PPT1 and PPT2 have an identical sequence, TGGGTGGTA, which is rather different from the 18 to 23 nucleotides long retroviral polypurine tract (Chen \& Barker, 1984). From the analysis of the effect of mutations introduced in PPT1 and PPT2 on the synthesis of DNA intermediates in Ty1 VLPs, direct evidence is provided here that the sequence TGGGTGGTA is required for the priming of Ty1 DNA plus-strands synthesis. Our results also suggest that the $T$ residue at the 5 ' end is not a crucial determinant for the PPT function. Moreover, inactivation of cDNA synthesis from PPT1 abolishes Ty1 transposition whereas inactivation of priming from PPT2 has no effect on transposition.

A consequence of the presence of two PPTs is that unintegrated Ty1 DNA exists as discrete plus-strand fragments at some time during replication. Three such fragments have been identified in the cDNA extracted from wild-type Ty1 VLPs: the plus-strand strong-stop cDNA initiated at PPT1, a downstream fragment initiated at PPT2 and an upstream fragment spanning the 5'-terminal part of Ty1 and a portion of the TyB gene (Pochart et al., 1993b). To understand the mechanism of plus-strand cDNA termination we have characterized the $3^{\prime}$ ends of the DNA plus-strand fragments synthesized in Ty1 VLPs. We show that the upstream fragment terminates predominantly 30 to 50 nucleotides downstream of the central PPT2 region. A similar termination step at the centre of the HIV-1 genome has been reported recently (Charneau et al., 1994). Thus, in Ty1 as in HIV-1, the upstream fragment terminates after strand displacement of the downstream fragment initiated at the internal PPT. The 3' end of the downstream fragment initiated at PPT2 and the 3' end of the plus-strand strong-stop cDNA were also investigated. We find that most of the plus-strand strong-stop cDNA fragments bear a copy of the PBS and that the downstream fragments terminate at the DNA-tRNA border. Our results are compatible with the accepted model for reverse transcription of retroviral genomic RNA. The implication of recent results (Lauermann \& Boeke, 1994) showing that the primer tRNA sequence is not inherited during Ty1 retrotransposition will be discussed.

\section{Results}

\section{Identification of plus-strand primers}

To identify the primers for plus-strand Ty 1 cDNA synthesis, specific nucleotide changes were introduced in the two putative PPT sequences TGGGTGGTA located immediately adjacent to the upstream $\mathrm{U} 3$ region (PPT1) and within the pol gene (PPT2). Details of these mutations are shown in Figure 1. PPT2 mutant 73 bears two nucleotide changes, $\mathrm{T}$ to $C$ and $G$ to $C$ at position -1 and +2 , respectively, that leave intact the protein coding sequence. PPT2 mutant 74 carries an additional $G$ to $C$ change at position +7 that leads to the replacement of a glycine by an alanine residue in the amino acid sequence of integrase (Monokian et al., 1994). PPT1 mutant 98 carries $\mathrm{G}$ to $\mathrm{C}$ mutations at positions +2 and +7 and an $\mathrm{A}$ to $\mathrm{C}$ mutation at position -1 . PPT1/2 mutant 74-98 carries mutations at positions $-1,+2$ and +7 in PPT1 and PPT2. In addition, a PPT1 mutant carrying a single $\mathrm{T}$ to $\mathrm{C}$ nucleotide change at position +1 was constructed (mutant 99). Indeed, two replicas of the PPT sequence with a $C$ residue at position +1 are found in the Ty1 sequence and do not seem to function as plus-strand priming sites. PPT1 mutant 99 was used to test the possibility that the $\mathrm{T}$ residue at position +1 is a determinant of the PPT function.

The PPT mutations were incorporated in a Ty1-neo element fused to the GAL1 promoter and subcloned in the pJEF 1105 multicopy plasmid (Chapman et al., 1992). The pJEF 1105 wild-type and mutant plasmids were transformed into the spt3 yeast strain AGY9 to minimize the amount of cDNA reverse transcripts specified by endogenous Ty1 elements (see Materials and Methods). The transformed yeast cells were grown under conditions that induce intracellular VLP formation. The cDNA extracted from the purified VLPs was examined by Southern blot analysis using a probe of minus polarity specific of the $\mathrm{R}$ region (Figure 2). In cells containing the wild-type Ty1-neo element two major fragments of $0.345 \mathrm{~kb}$ and $3.0 \mathrm{~kb}$ and a minor fragment of about $1.0 \mathrm{~kb}$ were detected (Figure 2B, lane $\mathrm{W}$ ). The $0.345 \mathrm{~kb}$ fragment is the plus-strand strong-stop cDNA initiated at PPT1. The $3.0 \mathrm{~kb}$ fragment which was also revealed by a neo probe (not shown) is the downstream fragment initiated at PPT2 spanning the 3 ' half of the TyB gene, the neo gene and the 3' LTR. The minor $1.0 \mathrm{~kb}$ fragment has not been identified. On overexposure of the autoradiogram the full length plus-strand of $6.9 \mathrm{~kb}$ and a $3.8 \mathrm{~kb}$ upstream fragment spanning the 5'-terminal part of Ty1 and a portion of the TyB gene were also revealed by the R-specific probe. The low amount of $6.9 \mathrm{~kb}$ and $3.8 \mathrm{~kb}$ strands indicates that plus-strand transfer is 
A

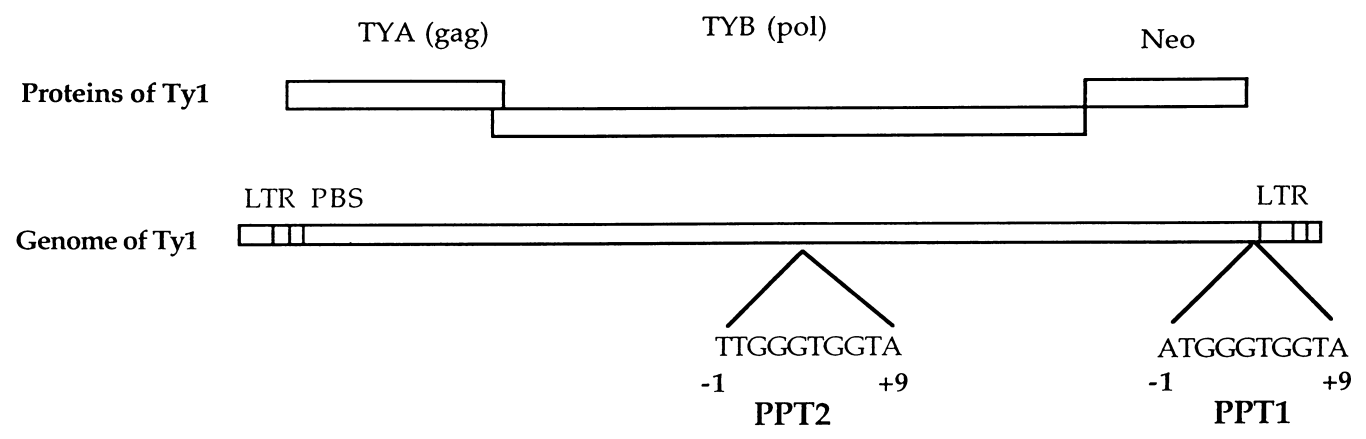

B

P P T 2

$\begin{array}{rlllllllll} & \text { asn } & \text { ser } & \text { ser } & \text { leu } & \text { gly } & \text { gly } & \text { ile } & \text { gly } & \text { asp } \\ \text { W T } & \text { AAT } & \text { TCC } & \text { AGT TTG } & \text { GGT } & \text { GGT } & \text { AAT } & \text { GGT } & \text { GAC } \\ & \text { asn } & \text { ser } & \text { ser leu } & \text { gly } & \text { gly } & \text { ile } & \text { gly } & \text { asp } \\ \mathbf{7 3} & \text { AAT } & \text { TCC } & \text { AGT } & \text { CTC } & \text { GGT } & \text { GGT } & \text { AAT } & \text { GGT } & \text { GAC } \\ & \text { asn } & \text { ser } & \text { ser leu } & \text { gly } & \text { ala } & \text { ile } & \text { gly } & \text { asp } \\ \mathbf{7 4} & \text { AAT } & \text { TCC } & \text { AGT } & \text { CTC } & \text { GGT } & \text { GCT } & \text { AAT } & \text { GGT } & \text { GAC }\end{array}$

PP T 1

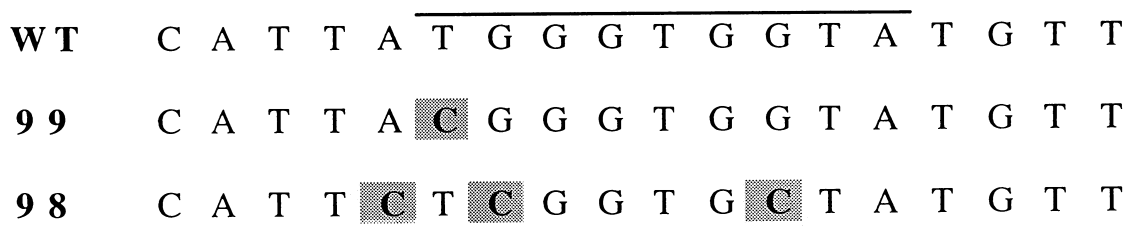

Figure 1. A, Positions and sequences of the two PPTs in the DNA genome of Ty1-neo with respect to the TyA (gag), TyB (pol) and neo sequences. Position -1 of PPT1 and PPT2 correspond to position 5576 and 3782 of the Ty1-H3 sequence, respectively (Boeke et al., 1988a). B, Details of the mutations introduced in PPT1 and PPT2. The amino acid sequence of the PPT2 region is indicated. In PPT2 mutant 74, a GGT codon is changed into GCT leading to the replacement of a glycine by an alanine residue. PPT1 is in the 3 ' non-coding region of the Ty1 sequence.

inefficient as previously observed in retroviral systems (Lee \& Coffin, 1991).

The Southern blot shown in Figure 2 reveals that mutations in the PPT sequences affect plus-strand cDNA synthesis. The synthesis of the $3.0 \mathrm{~kb}$ fragment is strongly reduced in PPT2 mutant 73 and is abolished in PPT2 mutant 74. Mutations introduced in the PPT1 sequence (mutant 98) inhibit the synthesis of the $0.345 \mathrm{~kb}$ fragment. In the double mutant PPT1/2 (74-98) the syntheses of the $0.345 \mathrm{~kb}$ and $3.0 \mathrm{~kb}$ fragments are both inhibited. In contrast, the $\mathrm{T}$ to $\mathrm{C}$ nucleotide change introduced at position +1 in PPT1 mutant 99 does not reduce the intensity of the signal given by the 0.345 fragment, indicating that this $\mathrm{T}$ residue is not important for priming from PPT1.

\section{Identification of double-stranded Ty1 DNA molecules synthesized in wild-type and PPT mutant VLPs}

Effect of PPT mutations on the synthesis of double-stranded Ty1 DNA was analysed by characterization of the restriction fragments derived from DNA molecules produced in wild-type and mutant VLPs. The expected size of full length linear double-stranded reverse transcript from the Ty1-neo element is $6.9 \mathrm{kbp}$. HpaI digestion of this full length 

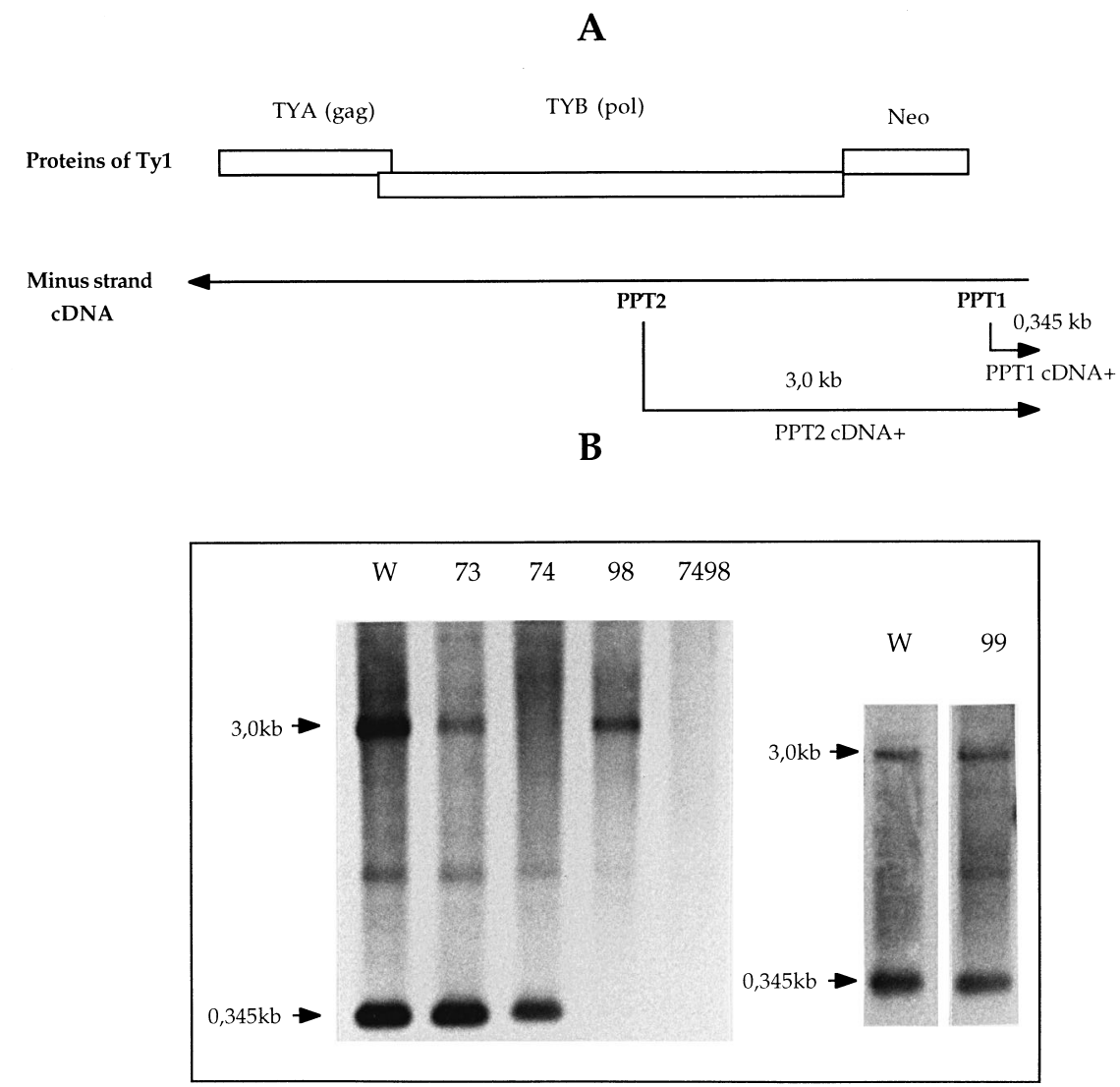

Figure 2. Analysis of the plus-strand DNA in Ty1-neo VLPs. A, The positions of the two origins of plus-strand DNA synthesis are indicated. PPT1 generates the $0.345 \mathrm{~kb}$ plus-strand strong-stop cDNA and PPT2 a $3.0 \mathrm{~kb}$ plus-strand fragment spanning the 3' half of the TyB gene, the neo gene and the 3' non-coding region of the Ty1 sequence. B, Plus-strand DNA extracted from VLPs from the spt3 yeast strain AGY9 containing wild-type (W) or PPT mutant $(73,74,98,99$ or 74-98) pJEF1105 plasmids was separated by electrophoresis on a $1 \%$ agarose gel. DNA was formamide-denatured prior to electrophoresis. Following transfer to nitrocellulose, the DNA fragments were detected using a radiolabelled minus-strand probe specific for the $\mathrm{R}$ region.

linear duplex should generate a $0.82 \mathrm{kbp}$ fragment from the 5 ' end of the molecules (Figure $3 \mathrm{~A}$ ). In the absence of strand transfer, the linear DNA molecule synthesized should be double-stranded only in the 3'-terminal part of the molecule between the PPT and the 3 ' end of the minusstrand cDNA and the $0.82 \mathrm{kbp}$ fragment should not be produced upon digestion with HpaI. A Southern blot of the HpaI digested DNA extracted from wild-type and PPT mutant VLPs was hybridized with a full-length Ty1 element probe (Figure 3B). As expected the $0.82 \mathrm{kbp}$ fragment was present in the DNA extracted from wild-type VLPs. It was also present in the DNA extracted from PPT2 mutant 74 but was not detected in the DNA from PPT1 mutant 98. The possibility exists that the $3.0 \mathrm{~kb}$ plus-strand fragment initiated at PPT2 in mutant 98 is transferred at the 3 ' end of the minus-strand cDNA. Minus-strand DNA synthesis could resume and duplicate the transferred $3.0 \mathrm{~kb}$ fragment. In that case a $3.5 \mathrm{kbp}$ fragment would be expected upon digestion with HpaI. Such a fragment is not observed in the autoradiogram of HpaI digested DNA from PPT1 mutant 98 VLPs. We therefore conclude that linear DNA molecules of the PPT1 mutant 98 are not double-stranded in the 5'-terminal part of the molecules indicating that strand transfer does not occur when PPT1 is not functional.

Double-strandedness of the $3^{\prime}$ end of the linear DNA molecules was checked by digestion with XhoI, which should generate a $1.3 \mathrm{kbp}$ fragment from the 3'-terminal part of the DNA (Figure 3A). As shown in Figure 3B the $1.3 \mathrm{kbp}$ fragment released by XhoI digestion was detected in DNA from wild-type as well as from PPT1 and PPT2 mutant VLPs. The presence of a $1.3 \mathrm{kbp}$ fragment was expected for control wild-type VLPs. It was also expected for the PPT1 mutant 98 since plus-strand cDNA initiated at PPT2 spans the XhoI site located in the neo gene. In the case of PPT2 mutant 74 in which plus-strand cDNA is not initiated at PPT2, the presence of the $1.3 \mathrm{kbp}$ fragment can be explained only if full length minus-strand cDNA has been copied after transfer of the strong-stop plus-strand cDNA. This result shows that initiation of plus-strand synthesis at PPT2 is not required for the synthesis of full-length doublestranded DNA.

We also checked for the presence of doublestranded one- or two-LTR circular forms of Ty1 DNA 
A
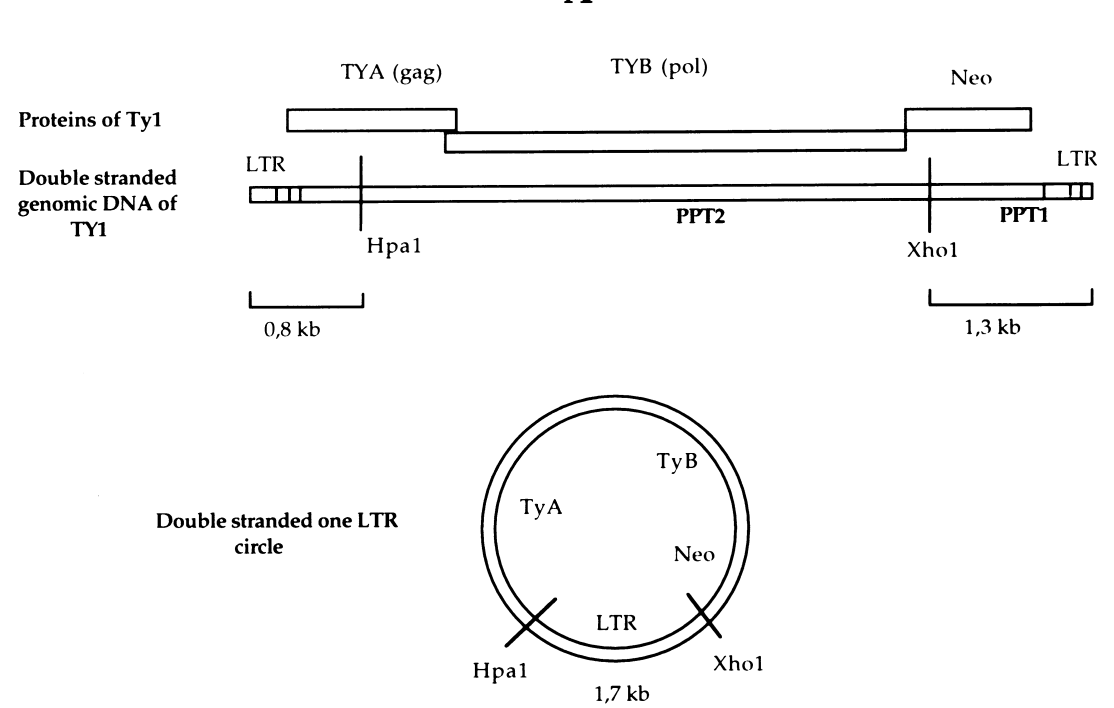

B

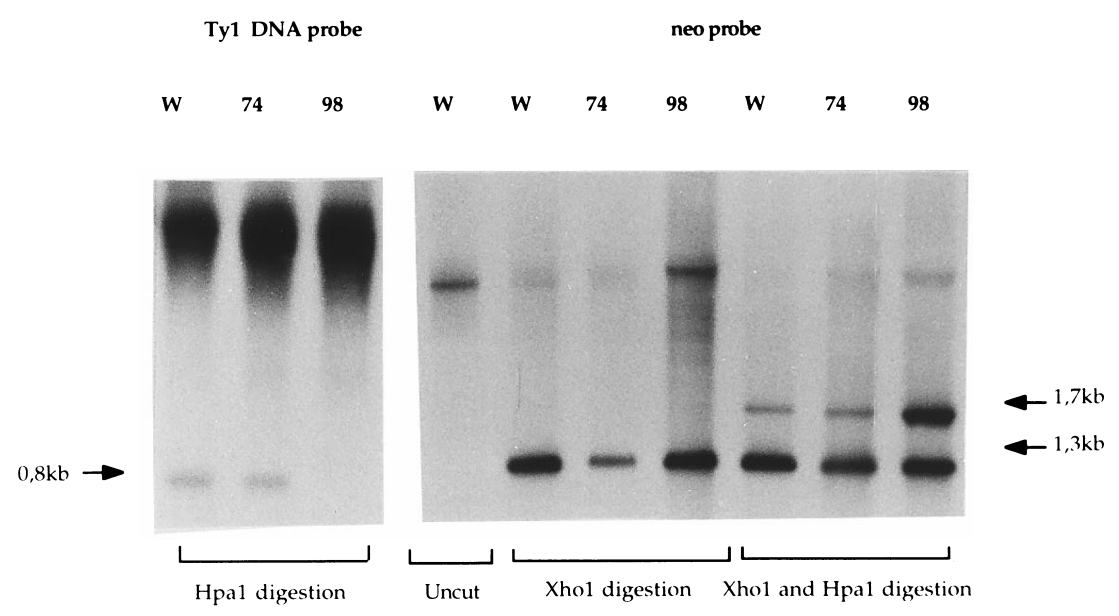

Figure 3. Analysis of double-stranded DNA in Ty1-VLPs. A, Maps of the linear and one-LTR circular forms of Ty1-neo DNA. The lengths of the fragments released by HpaI, XhoI or combined HpaI plus XhoI digestions are indicated. B, DNA extracted from VLPs was separated on a 1\% agarose gel after digestion with HpaI, XhoI or HpaI plus XhoI. Following transfer to nitrocellulose, the HpaI DNA fragments were detected using a Ty1 DNA full-length probe. The fragments generated by XhoI or HpaI plus XhoI digestion were detected using a neo probe.

by double digestion with $H p a \mathrm{I}$ and XhoI and hybridization with a neo probe. The expected product resulting from the cleavage of a two-LTR circle would be a $2.1 \mathrm{kbp}$ fragment generated by the ligation of the $5^{\prime}$ end $0.8 \mathrm{kbp}$ fragment to the $3^{\prime}$ end $1.3 \mathrm{kbp}$ fragment. A fragment of $1.7 \mathrm{kbp}$ would be released from a one-LTR circle (Figure 3A). As shown in Figure 3B, only $1.7 \mathrm{kbp}$ fragments were detected in the DNA pattern of the wild-type VLPs and PPT1 or PPT2 mutant VLPs. The $2.1 \mathrm{kbp}$ fragment was not detected indicating that two-LTR circles were not synthesized or synthesized in very low amount. A similar experiment performed by Eichinger \& Boeke (1988) also showed that the great majority of circular forms present in DNA extracted from wild-type Ty1-H3 VLPs contains one LTR. The formation of closed one-LTR circles implies that the synthesis of the minus-strand does not progress through the hybrid region formed between plusstrand strong-stop DNA and its donor template but that the synthesis of the plus-strand continues using the minus-strand as template (Figure 6). Quantification of the bands (Figure 3B) indicates that about $45 \%$ of DNA from PPT1 mutant VLPs and $17 \%$ of DNA from wild-type and PPT 2 mutant VLPs are present as one-LTR circles. The large amount of one-LTR circles detected in DNA from PPT1 mutant VLPs would be due to the fact that these forms are the only possible end-products of PPT1 mutants. 
A

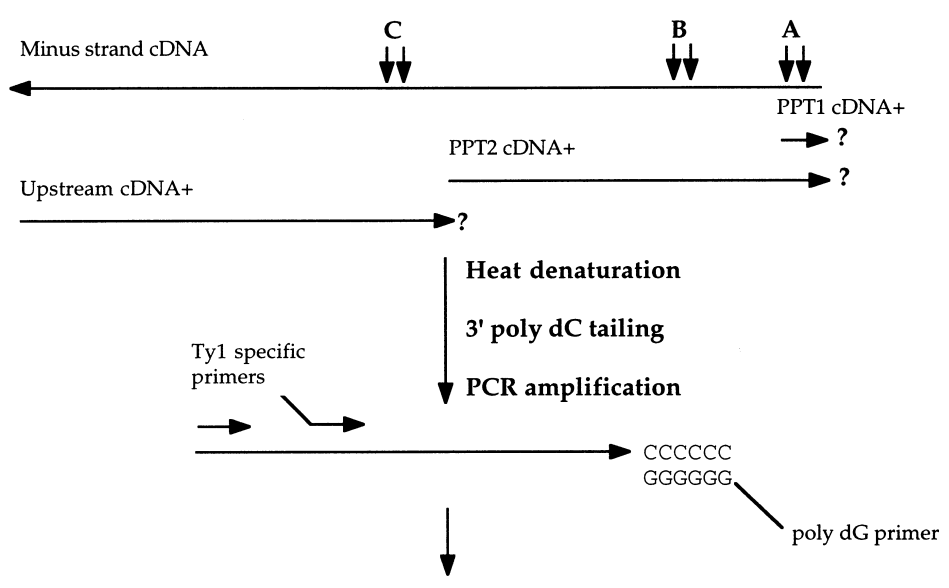

Subclone, sequence.

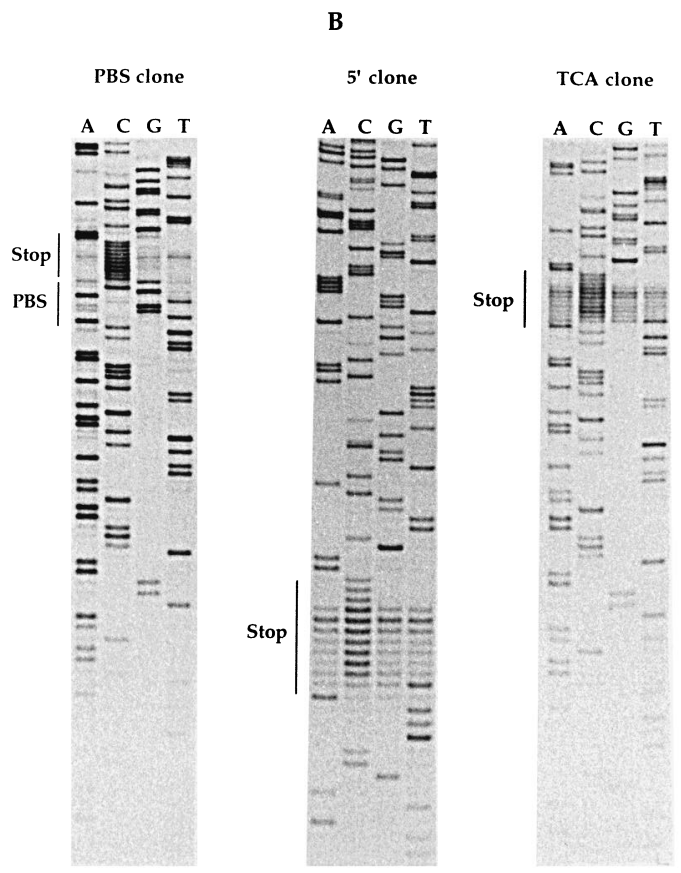

Figure 4. A, Amplification, cloning and sequencing of the 3' ends of the plus-strand DNA fragments. The DNA fragments from purified VLPs were poly $(\mathrm{dC})$-tailed by terminal transferase and amplified by two rounds of PCR amplification using nested Ty1-specific primers and a poly $(\mathrm{dG})$ primer complementary to the added poly $(\mathrm{dC})$ tail. The positions of the three combinations of nested primers A, B and C are indicated by arrows. B, Sequence analysis of typical amplification products obtained with combination A of nested primers. In the PBS clone, the PBS sequence has been reverse transcribed. In the TCA clone (T, $C$ and A being the three last nucleotides of the Ty1 sequence), reverse transcription has stopped at the DNA-tRNA border. In the 5' clone, reverse transcription has stopped before reaching the 3 ' end of the minus-strand DNA.

\section{Characterization of the 3' ends of Ty1 cDNA plus strands}

The anchored PCR technique (Charneau et al., 1994) was applied to selectively amplify the $3^{\prime}$ extremities of the plus-strand cDNA fragments and to map the 3' ends at the nucleotide level (Figure 4A). The cDNA fragments extracted from purified VLPs were poly $(\mathrm{dC})$-tailed using terminal transferase and selectively amplified by two rounds of PCR amplification. The PCR products were subcloned, sequenced and the 3 ' end of the amplified fragments was deduced from the position of the added poly $(\mathrm{dC})$-tail in the Ty1 sequence (Figure $4 \mathrm{~B})$. Three combinations (A, B and C) of nested primers were used to amplify the $3^{\prime}$ extremities of plus-strand cDNA fragments. Combination A amplifies the strong-stop plus-strand cDNA initiated at PPT1 (PPT1 cDNA+ in Figure 4A) and the downstream $3.0 \mathrm{~kb}$ fragment initiated at PPT2 (PPT2 cDNA+ in 
Table 1. Termination of DNA fragments amplified by combination A and B of nested primers

\begin{tabular}{|c|c|c|c|c|c|}
\hline & $\begin{array}{c}\text { Primer } \\
\text { combination }\end{array}$ & Ty1 element & 5 ' fragments & $\begin{array}{c}\text { TCA } \\
\text { fragments }\end{array}$ & $\begin{array}{c}\text { PBS } \\
\text { fragments }\end{array}$ \\
\hline I & A & W.T. & 2 & 6 & 11 \\
\hline II & B & W.T. & 1 & 11 & 1 \\
\hline III & A & W.T. 0.345 & 1 & - & 7 \\
\hline IV & B & Mutant 98 & 1 & - & 17 \\
\hline
\end{tabular}

Figure 4A). The full length plus-strand cDNA should also be amplified by combination A but is in very small amount compared to the $0.345 \mathrm{~kb}$ and $3.0 \mathrm{~kb}$ fragments.

Combination B amplifies the downstream $3.0 \mathrm{~kb}$ fragment initiated at PPT2 and the small amount of full length plus-strand. Combination $\mathrm{C}$ amplifies the $3^{\prime}$ extremities of the upstream $3.8 \mathrm{~kb}$ fragment (upstream cDNA+ in Figure 4A).

\section{3' Ends of the strong-stop plus-strand cDNA initiated at PPT1 and of the downstream $3.0 \mathrm{~kb}$ cDNA initiated at PPT2}

It is generally accepted that strand transfer of the strong-stop plus-strand DNA occurs after reverse transcriptase has reached the end of the PBS sequence in the tRNA primer covalently joined to the minus-strand DNA. Recent results of Lauermann \& Boeke (1994) indicating that the tRNA sequence is not inherited by progeny Ty1 elements during reverse transcription are inconsistent with this model. Previous hybridization experiments indicated that the tRNA sequence corresponding to the PBS was reverse-transcribed during plus-strand synthesis in vivo (Pochart et al., 1993b; Müller et al., 1991). It was therefore important to use a more accurate method to characterize the 3 ' ends of the plus-strand cDNA fragments. We first analysed the extremities of wild-type plus-strand cDNA using combination A of nested primers which amplifies the ends of the $0.345 \mathrm{~kb}$ and $3.0 \mathrm{~kb}$ fragments and the small amount of full length plus-strand DNA. As shown in Table 1 line I, more than half of the molecules bear a copy of the PBS (PBS fragments). The other molecules end at the DNA-tRNA border (TCA fragments: T, C and A being the three last nucleotides of the Ty1 sequence) or are even shorter ( 5 ' fragments). To analyse the ends of the strong-stop plus-strand cDNA separately, the $0.345 \mathrm{~kb}$ fragment was recovered after separation on an agarose gel and amplified using combination A of nested primers. We find that the majority of the $0.345 \mathrm{~kb}$ fragments bear a copy of the PBS (Table 1 line III). By using combination B of nested primers the ends of the $3.0 \mathrm{~kb}$ fragments were also analysed separately. We find that most of the $3.0 \mathrm{~kb}$ fragments terminate at the DNA-tRNA border (Table 1 line II). The fact that the $3.0 \mathrm{~kb}$ fragments are not terminated by a copy of the PBS implies that this fragment is extended toward the end of the minus-strand template DNA after completion of the $0.345 \mathrm{~kb}$ strong-stop plus-strand and removal by $\mathrm{RNaseH}$ of the tRNA molecule covalently joined to the minus-strand DNA template. A prediction of this observation is that the $3.0 \mathrm{~kb}$ fragment of PPT1 mutant 98 in which the synthesis of the $0.345 \mathrm{~kb}$ fragment is abolished should bear the PBS sequence since the tRNA attached to the minus-strand DNA template cannot be removed before completion of the $3.0 \mathrm{~kb}$ fragment synthesis. We find indeed that, in the absence of plus-strand strong-stop cDNA synthesis in PPT1 mutant 98, the majority of the $3.0 \mathrm{~kb}$ fragments bears a PBS sequence (Table 1 line IV). We therefore conclude that, in vivo, the portion corresponding to the PBS of the tRNA molecule covalently joined to the minus-strand DNA template is copied.

\section{Extremities of the upstream $3.8 \mathrm{~kb}$ plus-strand cDNA}

The upstream $3.8 \mathrm{~kb}$ plus-strand DNA is generated by the extension of the plus-strand strong-stop cDNA initiated at PPT1 after it has been transferred to the $3^{\prime}$ end of the minus-strand cDNA. Hybridization experiments with Ty1 strand-specific probes have shown that this fragment spans the 5 ' terminal part of the Ty1 sequence, extends through part of the TyB gene and ends close to PPT2 (Pochart et al., 1993b). Its 3' extremity was mapped to check the possibility that a termination step of plus-strand cDNA synthesis could be associated with initiation of plus-strand DNA synthesis at PPT2 as observed during HIV-1 reverse transcription. The distribution of the $3^{\prime}$ ends of the $3.8 \mathrm{~kb}$ plus-strand fragment is detailed in Figure 5. Most of the 3' ends (18 out of 21) are found downstream of PPT2 indicating that there is an overlap between the upstream $3.8 \mathrm{~kb}$ plus-strand DNA fragment and the downstream $3.0 \mathrm{~kb}$ fragment initiated at PPT2. A similar situation was observed with unintegrated HIV-1 DNA molecules for which discrete upstream and downstream segments have been identified. It was shown that the upstream segment terminates after a 88 or 98 nucleotides strand displacement of the downstream segment (Charneau et al., 1994). In the case of Ty1 the distribution of the 3' ends extends over 130 nucleotides but $30 \%$ are clustered 30 to 50 nucleotides downstream of PPT2 in an A + T-rich 


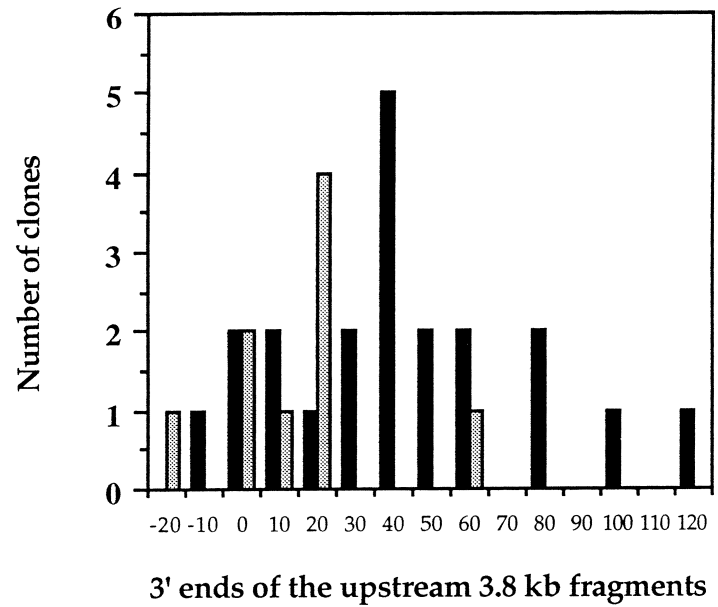

Figure 5. Distribution of the 3' ends of the DNA fragments from wild-type and mutant 74 VLPs amplified by combination $C$ of nested primers $(3.8 \mathrm{~kb}$ upstream fragments). The scale at the bottom of the panel indicates the distance (in nucleotides) from position 1 of PPT2. Wild-type VLPs; 㬂, mutant 74 VLPs.

region. As for HIV-1, this relatively narrow distribution of the observed stops of the Ty1 upstream fragments can be interpreted as an indication that a sequence-specific termination mechanism may be instrumental in blocking the progression of the $3.8 \mathrm{~kb}$ plus-strand DNA beyond PPT2. This hypothesis was confirmed by mapping the 3 ' ends of the $3.8 \mathrm{~kb}$ fragment from PPT2 mutant VLPs (mutant 74) in which the downstream $3.0 \mathrm{~kb}$ fragment is not synthesized. As illustrated in Figure 5 the distribution of the $3.8 \mathrm{~kb}$ fragment 3 ' ends of mutant 74 is very similar to that of wild-type elements showing clearly that termination is not only due to a limited capacity of the upstream $3.8 \mathrm{~kb}$ fragment to displace the downstream fragment initiated at PPT2 but must also lie in the sequence or structure of the termination region.

\section{Effect of PPT mutations on transposition}

Transposition frequency of the Ty1-neo elements can be quantified in vivo by detecting the G418-resistance phenotype of plasmid-free yeast cells which have undergone transposition. As shown in Table 2, the level of transposition of the two PPT2 mutant Ty 1 elements 73 and 74 is very similar and close to that of the wild-type Ty1 element. Since the synthesis of the $3.0 \mathrm{~kb}$ plus-strand is weakened in PPT2 mutant 73 VLPs and abolished in PPT2 mutant 74 VLPs, we conclude that the plus-strand fragment initiated at PPT2 is not required for Ty1 transposition.

In contrast, mutations in PPT1 that abolish plus-strand strong-stop cDNA synthesis also render Ty1 elements defective for transposition. Partially double-stranded linear DNA molecules and one-LTR circles detected in VLPs from PPT1 mutant 98 are therefore abortive products of Ty1 replication and cannot be used as transposition intermediates. As expected, a Ty1 mutant element bearing a $\mathrm{T}$ to $\mathrm{C}$ change at position +1 of PPT1 (mutant 99) in which plus-strand strong-stop DNA synthesis was not impaired shows a transposition frequency very close to that of the wild-type element.

\section{Discussion}

\section{The PPT sequence GGGTGGTA is required for initiation of plus-strand cDNA synthesis in Ty1}

During Ty1 reverse transcription, plus-strand synthesis of retrotransposon cDNA is initiated at two sites located at the upstream boundary of the 3' LTR and near the middle of the pol gene in the integrase coding sequence. The two putative primers for plus-strand cDNA synthesis, PPT1 and PPT2, have an identical sequence, TGGGTGGTA. We have used site-directed mutagenesis to demonstrate that this sequence is required for plus-strand priming. By analysis of the DNA fragments present in mutant VLPs we show that specific nucleotide changes introduced in PPT1 or PPT2 diminish or abolish the amount of plus-strand initiated at the mutated site. We also show that the $T$ residue at position +1 of the primer is not an absolute requirement for PPT function suggesting that the sequence GGGTGGTA might be sufficient to prime plus-strand DNA synthesis. The GGGTGGTA sequence used as plus-strand primer by Ty1 is a rather limited polypurine tract when compared to that of retroviruses. We have examined the putative plus-strand

Table 2. Transposition frequencies of wild-type and PPT mutant Ty1 elements (assayed in yeast strain MW16)

\begin{tabular}{|c|c|c|c|c|}
\hline & \multicolumn{3}{|c|}{$\mathrm{Neo}^{\mathrm{r}} \mathrm{Ura}^{-}$colonies/Ura${ }^{-}$colonies } & \multirow[t]{2}{*}{$\begin{array}{l}\text { Transposition } \\
\text { frequencies }(\%)\end{array}$} \\
\hline & Exp. 1 & Exp. 2 & Exp. 3 & \\
\hline Wild-type & $137 / 355$ & $101 / 464$ & $237 / 925$ & 27.2 \\
\hline PPT2 mutant 73 & $59 / 317$ & $72 / 606$ & $300 / 1336$ & 19.1 \\
\hline \multirow[t]{2}{*}{ PPT2 mutant 74} & $84 / 374$ & $68 / 527$ & $392 / 1545$ & 22.2 \\
\hline & Exp. 4 & Exp. 5 & & \\
\hline Wild-type & $385 / 704$ & $126 / 366$ & & 47.7 \\
\hline PPT1 mutant 99 & $315 / 824$ & $204 / 542$ & & 37.9 \\
\hline PPT1 mutant 98 & $12 / 625$ & $11 / 434$ & & 2.2 \\
\hline PPT1/2 mutant 74-98 & $3 / 708$ & & & 0.4 \\
\hline
\end{tabular}


priming sites of the other functional retroelements Ty2, Ty3 and Ty4 identified in Saccharomyces cerevisiae (Boeke et al., 1988a; Warmington et al., 1985; Hansen et al., 1988; Stucka et al., 1992). Ty2 is highly related to Ty1, it shares the same LTR sequence, a fairly similar protein sequence and possesses the GGGTGGTA sequence at comparable positions. Ty4 is a low copy number element which shows a genomic organization homologous to that found in Ty1 and Ty2 but diverges from these elements at the protein sequence level, the highest similarity being in the reverse transcriptase domain. The sequence of the putative plus-strand priming site of Ty4 is AAGGGAGCA, thus is richer in purine than the PPTs of Ty 1 or Ty 2 but shorter than the retroviral PPTs. In contrast Ty3, more related to some animal retroviruses than to Ty1 and Ty2 by its genomic organization and the amino acid sequence of its reverse transcriptase domain, possesses a putative plus-strand priming site comprising 14 consecutive purines.

The differences observed between the sequences used as plus-strand primers by various retroelements suggest that the specificity requirements of reverse transcriptase associated $\mathrm{RNaseH}$ activity is different from one system to another. It is also possible that the recognition signal for specific primer formation and elongation lies in the overall conformation of the PPT domain. The implication of regions outside of the Ty1 PPTs in plus-strand priming is suggested by the presence of two GGGTGGTA sequences located at positions 3344 and 3500 in the Ty1-H3 sequence (Boeke et al., 1998a) that do not seem to be used as primers. Therefore the GGGTGGTA sequence is not sufficient by itself to generate a plus-strand origin site although our mutagenesis experiments show that the integrity of the properly located sequence is an absolute requirement for plus-strand DNA synthesis.

\section{PPT1 is sufficient for Ty1 transposition}

We have examined the role of PPT mutations on transposition. A Ty1 mutant with an inactive PPT1 is defective for transposition. The fact that such a mutant does not produce full length double-stranded Ty1 DNA molecules but only partially doublestranded molecules and large amounts of one-LTR circles indicates that these molecules are not used as transposition intermediates. In contrast, inactivation of PPT2 has no significant effect on transposition. This is in agreement with the results of $\mathrm{Xu} \&$ Boeke (1990) who showed that large regions of Ty1 including PPT2 could be deleted without eliminating transposition, the functional gene products being supplied in trans from a helper Ty1 element defective for transposition. The lack of transposition defect of the PPT2 mutant Ty1 element indicates that the synthesis of plus-strand initiated at PPT2 is not important for Ty1 replication. Similar experiments done with HIV-1 show that the central PPT is not an absolute requirement for viral replication but confers a relative replication advantage to the virus (Charneau et al., 1992).

\section{Unintegrated linear Ty1 DNA includes a central plus-strand overlap}

A consequence of the presence of two plus-strand start sites is that Ty 1 plus-strand DNA exists as segments during the process of reverse transcription: in addition to the plus-strand strong-stop initiated at PPT1, a $3.0 \mathrm{~kb}$ downstream fragment and a $3.8 \mathrm{~kb}$ upstream fragment have been identified in Ty1 VLPs from pJEF 1105 transformed yeast cells. Primer extension experiments have shown that the $3.0 \mathrm{~kb}$ downstream fragment is initiated at PPT2 (Pochart et al., 1993b). The $3.8 \mathrm{~kb}$ upstream fragment results from the elongation of the plus-strand strong-stop cDNA initiated at PPT1 after its transfer to the $3^{\prime}$ end of the minus-strand. The length of the $3.8 \mathrm{~kb}$ fragment suggests that it may terminate close to the PPT2 sequence. The results of the 3' end mapping experiments reveal that this fragment is in fact elongated beyond the PPT2. Thus, the upstream and the downstream fragment overlap in the PPT2 region. Recent results have shown that unintegrated HIV-1 DNA also includes a central plus-strand overlap (Charneau et al., 1994). Linear DNA with a segmented plus-strand is the final product of HIV-1 reverse transcription whereas Ty1 VLPs contain detectable amounts of full-length plus-strand DNA (Pochart et al., 1993b). The Ty1 full-length strand could result either from ligation of the segments after removal of the overlapping sequence or by extension of a plus-strand fragment able to overcome the termination region (Figure 6). The latter possibility is supported by the fact that full-length DNA capable of transposition is synthesized by a PPT2 mutant element lacking a functional second plus-strand origin: the presence of $3.8 \mathrm{~kb}$ fragments in the PPT2 mutant suggests that DNA plus-strand synthesis could be temporarily interrupted or slowed down in the PPT2 region and then resume across the termination region to give a full-length strand.

\section{The PBS complementary sequence of the primer tRNA is efficiently reverse transcribed during plus-strand synthesis}

According to the models of retroviral reverse transcription, the second strand transfer involves complementary copies of the PBS. One copy of the PBS is formed when the PBS region of the genomic RNA is reverse transcribed during minus-strand synthesis. The complementary copy located at the 3' end of the plus-strand strong-stop DNA is generated by copying the portion corresponding to the PBS of the tRNA molecule covalently joined to the minus DNA template. Annealing of the two complementary sequences gives rise to a circular intermediate which allows completion of full-length two-LTR linear DNA. Our results, showing that the great majority of plus-strand strong-stop DNA molecules synthesized by Ty1 element bears a PBS sequence at their 3' end, 


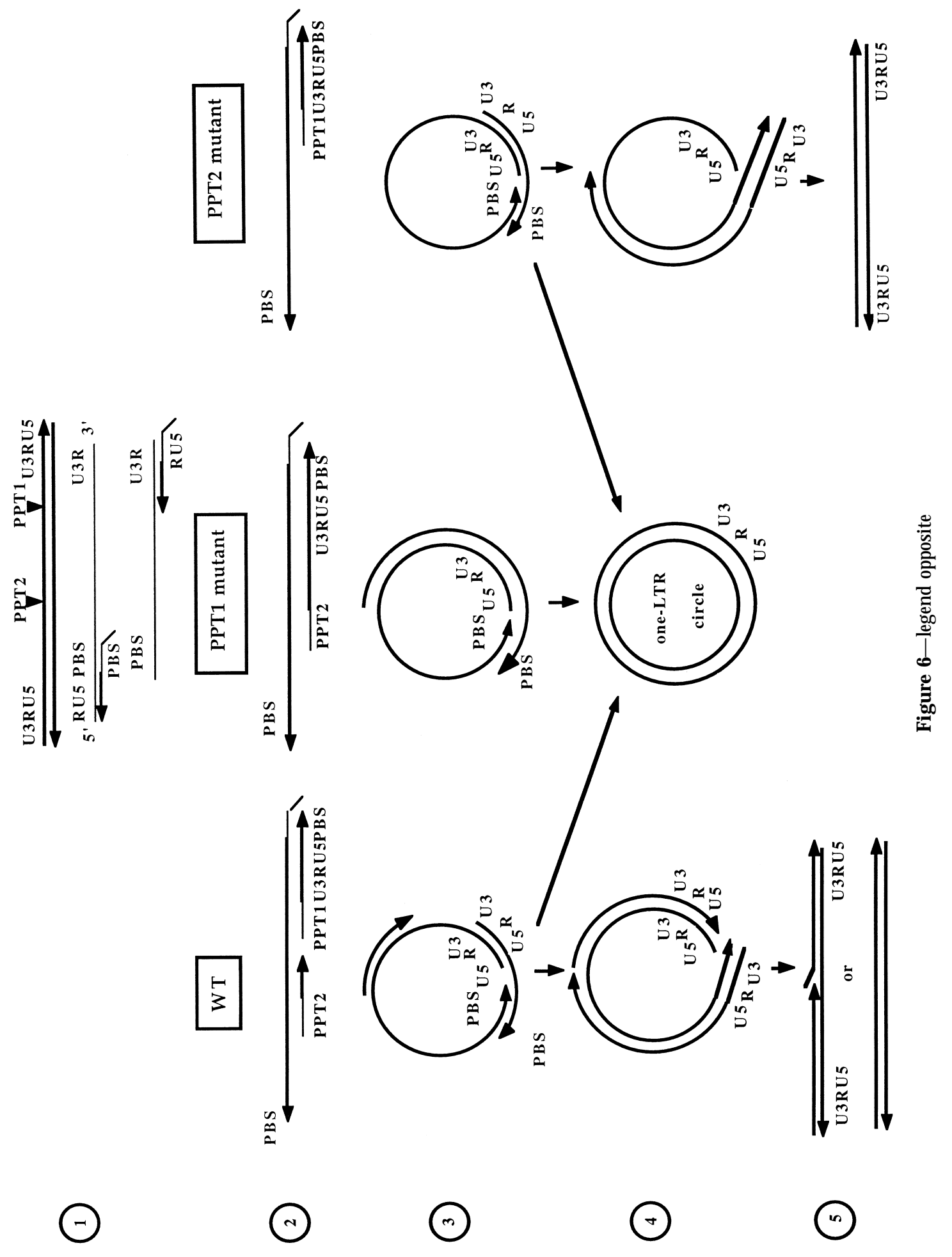


suggest that Ty1 second strand transfer could proceed as described for retroviruses. However, recent observations by Lauermann \& Boeke (1994) indicate that the primer tRNA sequence might not be inherited during Ty1 reverse transcription. Two possibilities have been proposed to explain this result: (1) the tRNA is not copied in the DNA molecules destined to be inherited or (2) the tRNA is copied but the tRNA-derived sequences are removed prior to further extension. Our results show that the PBS copy is detected at the end of the majority of plus-strand strong-stop cDNA molecules. On the other hand we also observe the presence of a few shorter plus-strand strong-stop cDNA molecules ending within the U5 region or at the tRNA-DNA boundary (Table 1). The possibility that these shorter molecules are the only productive intermediates for full-length two-LTR DNA molecules cannot be excluded and raises the question of how the second strand transfer occurs without annealing of the PBS complementary sequences. A model was proposed by Lauermann \& Boeke (1994) in which the minus-strand strong-stop cDNA is transferred to a second genomic RNA molecule that has the R-U5 or U5 sequence, generating a minus-strand cDNA molecule with the R-U5 or U5 sequence. In this model the less than full-length plus-strand strong-stop DNA could anneal to the complementary R-U5 or U5 sequence and would be dissociated from the 5 ' end of the minus-strand by the growing point of plus-strand initiated at PPT2. Our results show that the displacement of plus-strand strong-stop cDNA by the strand initiated at PPT2 is not necessary for the synthesis of full-length DNA since we find that the level of transposition of a PPT2 mutant for which the synthesis of the plus-strand DNA from PPT2 is abolished is similar to that of the wild-type Ty1 element. It is clear at this point that the mechanism of the second strand transfer is not well understood and that a model consistent with these different results remains to be elaborated.

\section{Concluding remarks}

Our in vivo analysis of the primer sites for plus-strand cDNA synthesis shows that the integrity of the 3' end PPT is critical for Ty1 replication. In contrast, synthesis of plus-strand primed by the internal PPT is not necessary for Ty 1 replication. This result raises the question of the biological role of a second origin of Ty 1 cDNA plus-strand synthesis. Initiation of cDNA plus-strand synthesis at multiple sites leading to incomplete segments is thought to contribute to retroviral recombination ( $\mathrm{Hu} \&$ Temin, 1990). It was proposed that during reverse transcription, single-stranded branches displaced from the minus-strand of cDNA copied from one genome in the virion might anneal to an homologous region of the minus-strand cDNA copied from the second RNA genome in the virion giving rise to a heteroduplex (Junghans et al., 1982). Although there is no direct evidence that two genomic RNA molecules are packaged into Ty1 VLPs, this assumption is supported by the result of genetic experiments suggesting that recombinant progeny elements derive from the reverse transcription of different RNA molecules (Boeke et al., 1985, 1986). The possibility that cDNA plus-strand displacement during Ty1 reverse transcription is involved in the generation of hybrid progeny elements is under investigation.

\section{Materials and Methods}

\section{Strains and plasmids}

The yeast strain AGY9 (Mat a, his 4-539, lys 2-801, leu $2 \Delta 1$, trp 1 $\Delta 63$, ura3-52, spt 3-202, GAL+) kindly provided by J. D. Boeke was used to minimize the amount of reverse transcript specified by endogenous Ty1 elements since transcription of genomic Ty1 elements is reduced 10- to 20 -fold in this strain whereas the transcription of GAL1 promoted elements is unaffected (Mathias et al., 1991). Plasmid pJEF 1105 (Boeke et al., 1988b) kindly provided by J. D. Boeke is a high copy number $(2 \mu \mathrm{m})$ plasmid marked

Figure 6. Proposed model for reverse transcription of wild-type and PPT mutant Ty1 elements. This Figure presents the overall flow of reverse transcription of the Ty1 element with emphasis on initiation of plus-strand synthesis at PPT1 and PPT2. For convenience, the complementary copies of the PBS have been annealed in the plus-strand transfer (step 3) as described in the retroviral model for reverse transcription. It is concievable that a different type of plus-strand transfer may occur but its mechanism is not known. Note that the drawings are not to scale. (1) The minus-strand strong-stop DNA is initiated at the tRNA primer annealed to the PBS site. The first strand transfer occurs via the repeated R sequence after degradation of the R-U5 region by RNase-H. (2) The DNA minus-strand elongated up to the PBS serves as template for DNA plus-strand synthesis after degradation of the RNA genome by RNaseH. DNA plus-strand synthesis is primed by RNaseH resistant fragments called PPTs. The 3' end PPT, called PPT1, primes the synthesis of plus-strand strong-stop DNA (wild-type and PPT2 mutant elements) whereas the internal PPT, called PPT2, primes the synthesis of a $3.0 \mathrm{~kb}$ plus-strand (wild-type and PPT1 mutant elements). (3) A circular intermediate is formed by annealing of the PBS complement at the 3' end of the minus-strand to the PBS copy located either at the 3' end of the plus-strand strong-stop DNA (wild-type and PPT2 mutant elements) or at the 3' end of the $3.0 \mathrm{~kb}$ strand (PPT1 mutant elements). (4) The 5' LTR is formed by strand displacement synthesis from the minus-strand 3' end (wild-type and PPT2 mutant elements). Plus-strand synthesis primed by the 3' end of plus-strand strong-stop DNA gives rise to $3.8 \mathrm{~kb}$ fragments. The arrest of DNA synthesis beyond the PPT2 site creates a plus-strand overlap in the case of the wild-type element. One-LTR circle is formed in the absence of displacement of the plus-strand DNA. (5) Continuous plus-strand is formed by DNA synthesis primed by the 3' end of the upstream $3.8 \mathrm{~kb}$ fragment overcoming the termination region and displacing the downstream fragment synthesized from PPT2. In the case of the wild-type element, another possibility is the ligation of fragments after the removal of the single-stranded tail. 
with URA3 containing a Ty1-neo element fused to the GAL1 promoter. The neo gene is inserted at the BglII site (position 5562) of the Ty1-H3 sequence (Boeke et al.,1988a). The yeast strain AGY9 was transformed either by wild-type plasmid pJEF 1105 or by this plasmid carrying the nucleotide changes described below. Upon galactose induction, cells transformed by these plasmids produce large amounts of VLPs. Growth conditions were as described (Pochart et al., 1993a).

\section{Analysis of Ty1-VLP DNA}

Ty1-VLPs were isolated on a sucrose step gradient using a method described by Eichinger \& Boeke (1988) with minor modifications (Pochart et al., 1993a). Extraction of DNA from VLPs, electrophoresis on agarose gels, blotting, and hybridization with 5'-labelled oligomer or DNA fragments were as described (Pochart et al., 1993b): 1\% (w/v) agarose gel in $50 \mathrm{mM}$ Tris-borate ( $\mathrm{pH} 8.3), 1 \mathrm{mM}$ EDTA were used to analyse single-stranded or doublestranded DNA fragments. To analyse single-stranded DNA, samples were denatured at $90^{\circ} \mathrm{C}$ in $90 \%$ formamide, $10 \mathrm{mM}$ Tris- $\mathrm{HCl}$ (pH 8.0), $1 \mathrm{mM}$ EDTA before loading on the gel. In these conditions the positions of the molecular weight markers indicate that DNA migrates as a function of molecular weight in the non-denaturing gel. The sequence of the oligonucleotide probe specific for the $\mathrm{R}$ region is derived from the DNA sequence of the Ty1H3 element (Boeke et al., 1988a); it is complementary to positions 251 to 270 or 5835 to 5854 of Ty1: 5'GGTATACAGAATATACTAGA3'. The XhoI-BamH1 fragment, position 3840 to 9440, of plasmid pGTyl-H3 was used as Ty1 full-length probe. The XhoI-HindIII fragment, position 6191 to 6711 , of plasmid pJEF 1105 was used as a neo probe. When necessary the gels were analysed with a Molecular Dynamics phosphoimager and the bands were quantified by direct computer analysis of the stored images.

\section{Poly(dC) tailing, anchored PCR and subcloning of the amplified products}

The poly $(\mathrm{dC})$ tailing and the anchored PCR was done as described by Charneau et al. (1994). Terminal transferase was used to add homopolymer tails to DNA fragments extracted from purified VLPs. The DNA was heatdenatured at $95^{\circ} \mathrm{C}$ for two minutes and poly(dC) tailing was achieved for 15 minutes at $37^{\circ} \mathrm{C}$ in a final volume of $20 \mu \mathrm{l}$ in the presence of 50 units of terminal transferase EC2.7.7.31 (Boehringer-Mannheim), 100 pmol dCTP, $0.75 \mathrm{mM} \mathrm{CoCl} 2,200 \mathrm{mM}$ potassium cacodylate, $25 \mathrm{mM}$ Tris-HC1 ( $\mathrm{pH} 6.6), 0.25 \mathrm{mg} / \mathrm{ml}$ bovine serum albumin. The reaction was stopped by EDTA and the DNA was phenol/chloroform purified and precipitated by ethanol.

The poly $(\mathrm{dC})$ tailed DNA fragments were subjected to two sequential rounds of anchored PCR amplification. The first PCR amplification was performed with a Ty1-specific primer and an oligo(dG) primer complementary to the added 3' poly $(\mathrm{dC})$ tail to enhance the specificity of amplification. A second round of PCR was performed using a nested Ty1-specific primer and the oligo(dG) primer. The reaction conditions were $50 \mathrm{mM} \mathrm{KCl}, 10 \mathrm{mM}$ Tris- $\mathrm{HCl}$ ( $\mathrm{pH} 8.4$ ), $0.1 \mathrm{mg} / \mathrm{ml}$ gelatin, $1.5 \mathrm{mM} \mathrm{MgCl}_{2}$, $0.8 \mathrm{mM}$ dNTPs, $16 \mathrm{ng} / \mu \mathrm{l}$ each of primers, $1 \mu \mathrm{l}$ DNA solution, in a final volume of $100 \mu \mathrm{l}$. PCR amplification was carried out for 35 cycles at $92^{\circ} \mathrm{C}$ for two minutes, $50^{\circ} \mathrm{C}$ for two minutes and $72^{\circ} \mathrm{C}$ for two minutes. The resulting PCR amplification products were extracted with phenol/chloroform and digested by EcoRI and HindIII. The digested products were ligated to EcoRI and HindIII cut pSL1180 phagemid and competent Escherichia coli cells were transformed. Small-scale phagemid DNA preparation of individual clones was used for double-stranded DNA sequencing.

\section{Oligonucleotide primers}

Oligonucleotide primers were synthesized using an Applied Biosystem 381 A DNA synthesizer. The following primers were used for the two rounds of anchored PCR: $3.8 \mathrm{~kb}$ fragment first round of PCR: primer 178 position 4178 to 4197 of pJEF 1105 5'-ATCGAATTCCGAGGAATCTATCATCGCTG-3' and poly(dG) primer 9924 5'-ATCGAAGCTTGGGGGGGGGG-3'; 3.8 kb fragment second round of PCR: primer 9915 position 4245 to 4262 of pJEF 1105 5'-ATCGAATTCGACCCATTTAAAGAACTC-3' and poly(dG) primer $9924.3 .0 \mathrm{~kb}$ fragment first round of PCR: primer 116 position 6819 to 6842 of pJEF 1105 5'-ATCGAATTCGTCGGAATCGCAGACCGATACC-3' and poly(dG) primer $9924.3 .0 \mathrm{~kb}$ fragment second round of PCR: primer 276 position 7000 to 7020 of pJEF 1105 5'-ATCGAATTCAACACTGGCAGAGCATTACGC-3' and poly(dG)primer $9924.0 .345 \mathrm{~kb}$ fragment first round of PCR: primer 274 position 7175 to 7193 of pJEF 1105 5'-ATCGGATCCTGGAAGCTGAAACGCAAGG-3' and poly(dG) primer $9924.0 .345 \mathrm{~kb}$ fragment second round of PCR: primer 275 position 7254 to 7273 of pJEF 1105 5'-ATCGAATTCAGAATTGTGTAGAATTGCAG-3' and poly(dG) primer 9924.

The following primers were used for mutagenesis of PPT1 and PPT2: primer 8698 (PPT1 mutant 98): 5'-ATTCCAACATAGCACCGAGAATGTAATAG-3' primer 8699 (PPT1 mutant 99) 5'-ATTCCAACATACCACCCGTAATGTAATAG-3' primer 1473 (PPT2 mutant 73) 5'-GTTGATAGTAGTATATGCATTAGAGTCACCAATACCACCGAGACTGGAATTAG-3' primer 1474 (PPT2 mutant 74) 5 ' - GTTGATAGTAGTATATGCATTAGAGTCACCAATAGCACCGAGACTGGAATTAG-3'.

\section{Construction of mutants}

Site-directed mutagenesis was performed as described by Kunkel (1985). A 3597 bp BamHI-KpnI fragment from pJEF 1105 was subcloned into phagemid pSL1190 and mutagenized. Following mutagenesis, the $3597 \mathrm{bp}$ Bam HIKpnI fragment was cloned back into pJEF 1105.

\section{Transposition assay}

The transposition assay was performed in yeast strain

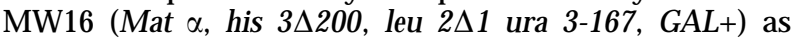
described by Chapman et al. (1992). Yeast strains harbouring the wild-type or mutant Ty1 elements on pJEF1105 were grown at $22^{\circ} \mathrm{C}$ for five days on yeast nitrogen base without amino acids (YNB) complemented with the required amino acids and containing $2 \%(\mathrm{w} / \mathrm{v})$ galactose. The cells were scraped, diluted in water and plated as single colonies to non-selective medium YPD to allow for plasmid loss. Following two days of growth at $30^{\circ} \mathrm{C}$ the plates were replica-plated to a YNB medium containing $1 \mathrm{mg}$ 5-fluoroorotic acid (5FOA) per $\mathrm{ml}, 50 \mu \mathrm{g}$ uracil $/ \mathrm{ml}$ and the required amino acids to select for cells that have lost the pJEF1105 plasmid containing the URA3 gene. After two days of growth at $30^{\circ} \mathrm{C}$ the plates were replicated to YPD medium containing $75 \mu \mathrm{g}$ of $\mathrm{G} 418$ per ml and to YNB medium containing the required amino acids and $2 \%(\mathrm{w} / \mathrm{v})$ glucose to identify colonies $\left(\mathrm{Neo}^{\mathrm{r}} \mathrm{Ura}^{-}\right)$that had undergone transposition of the Ty1-neo element. 


\section{Acknowledgements}

We thank Roland Chanet and Francis Fabre for discussions and encouragement. We are grateful to Professor G. Dirheimer for his constant interest in our work. This work was supported in part by grants from the Association pour la Recherche contre le Cancer (ARC) and from the Ligue Nationale Française contre le Cancer, Comité Départemental du Haut-Rhin.

\section{References}

Boeke, J. D. \& Sandmeyer, S. (1991). Yeast transposable elements. In The Molecular and Cellular Biology of the Yeast Saccharomyces cerevisiae (Broach, J. R., Jones, E. W. \& Pringle, J., eds), pp. 193-261, Cold Spring Harbor Laboratory Press, Cold Spring Harbor, NY.

Boeke, J. D., Garfinkel, D. J., Styles, C. A. \& Fink, G. R. (1985). Ty elements transpose through an RNA intermediate. Cell, 40, 491-500.

Boeke, J. D., Styles, C. A. \& Fink, G. R. (1986). Saccharomyces cerevisiae SPT3 gene is required for transposition and transpositional recombination of chromosomal Ty elements. Mol. Cell. Biol. 6, 3575-3581.

Boeke, J. D., Eichinger, D., Castrillon, D. \& Fink, G. R. (1988a). The Saccharomyces cerevisiae genome contains functional and nonfunctional copies of transposon Ty1. Mol. Cell. Biol. 8, 1432-1442.

Boeke, J. D., Xu, H. \& Fink, G. R. (1988b). A general method for the chromosomal amplification of genes in yeast. Science, 239, 280-282.

Chapman, K. B., Byström, A. S. \& Boeke, J. D. (1992). Initiator methionine tRNA is essential for Ty1 transposition in yeast. Proc. Natl Acad. Sci. USA, 89, 3236-3240.

Charneau, P. \& Clavel, F. (1991). A single-stranded gap in human immunodeficiency virus unintegrated linear DNA defined by a central copy of the polypurine tract. J. Virol. 65, 2415-2421.

Charneau, P., Alizon, M. \& Clavel, F. (1992). A second origin of DNA plus-strand synthesis is required for optimal immunodeficiency virus replication. J. Virol. 66, 2814-2820.

Charneau, P., Mirambeau, G., Roux, P., Paulous, S., Buc, H. \& Clavel, F. (1994). HIV-1 reverse transcription: a termination step at the center of the genome. J. Mol. Biol. 241, 651-662.

Chen, H. R. \& Barker, W. C. (1984). Nucleotide sequences of the retroviral long terminal repeats and their adjacent regions. Nucl. Acids Res. 12, 1767-1778.

Eichinger, D. J. \& Boeke, J. D. (1988). The DNA intermediate in yeast Ty1 element transposition copurifies with virus-like particles: cell-free Ty1 transposition. Cell, 54, 955-966.
Hansen, L. J., Chalker, D. L. \& Sandmeyer, S. B. (1988). Ty3, a yeast retrotransposon associated with tRNA genes, has homology to animal retroviruses. Mol. Cell. Biol. 8, 5245-5256.

Hu, W. S. \& Temin, H. M. (1990). Retroviral recombination and reverse transcription. Science, 250, 1227-1232.

Junghans, R. P., Boone, L. R. \& Skalka, A. M. (1982). Products of reverse transcription in avian retrovirus analyzed by electron microscopy. Cell, 30, 53-62.

Kunkel, T. A. (1985). Rapid and efficient site-specific mutagenesis without phenotypic selection. Proc. Natl Acad. Sci. USA, 82, 488-492.

Lauermann, V. \& Boeke, J. D. (1994). The primer tRNA sequence is not inherited during Ty1 retrotransposition. Proc. Natl Acad. Sci. USA, 91, 9847-9851.

Lee, Y. M. H. \& Coffin, J. M. (1991). Relationship of avian retrovirus DNA synthesis to integration in vitro. Mol. Cell. Biol. 11, 1419-1430.

Mathias, S. L., Scott, A. F., Kazazian, H. H. Jr, Boeke, J. D. \& Gabriel, A. (1991). Reverse transcriptase encoded by a human transposable element. Science, 254, 18081810.

Monokian, G. M., Braiterman, L. T. \& Boeke, J. D. (1994). In-frame linker insertion mutagenesis of yeast transposon Ty1: mutations transposition and dominance. Gene, 139, 9-18.

Müller, F., Laufer, W., Pott, U. \& Ciriacy, M. (1991). Characterization of products of Ty1-mediated reverse transcription in Saccharomyces cerevisiae. Mol. Gen. Genet. 226, 145-153.

Pochart, P., Agoutin, B., Fix, C., Keith, G. \& Heyman, T. (1993a). A very porly expressed tRNA ${ }^{\text {Ser }}$ is highly concentrated together with replication primer initiator tRNA $^{\text {Met }}$ in the yeast Ty1 virus-like particles. Nucl. Acids Res. 21, 1517-1521.

Pochart, P., Agoutin, B., Rousset, S., Chanet, R., Doroszkiewicz, V. \& Heyman, T. (1993b). Biochemical and electron microscope analysis of the DNA reverse transcripts present in the virus-like particles of the yeast transposon Ty1. Identification of a second origin of Ty1 DNA plus strand synthesis. Nucl. Acids Res. 21, 3513-3520.

Stucka, R., Schwarzlose, C., Lochmüller, H., Häcker, U. \& Feldmann, H., (1992). Molecular analysis of the yeast Ty4 element: homology with Ty1, copia, and plant retrotransposons. Gene, 122, 119-128.

Warmington, J. R., Waring, R. B., Newlon, C. S., Indge, K. J. \& Oliver, S. G. (1985). Nucleotide sequence characterization of Ty 1-17, a class II transposon from yeast. Nucl. Acids Res. 13, 6679-6693.

Xu, H. \& Boeke, J. D. (1990). Localization of sequences required in cis for yeast Ty1 element transposition near the long terminal repeats: analysis of mini Ty1 elements. Mol. Cell. Biol. 10, 2695-2700.

Edited by M. Yaniv 